\title{
Electron Correlation Microscopy for Studying Fluctuating Systems In Situ
}

\author{
Debaditya Chatterjee, Pei Zhang, and Paul M. Voyles*
}

Department of Materials Science \& Engineering, University of Wisconsin-Madison, Madison, WI, USA. * Corresponding author: paul.voyles@wisc.edu

Many physical systems exhibit fluctuations in equilibrium at non-zero temperature. Examples include fluctuating domains in ferroelectrics or ferromagnets without a external field, motions of atoms in a liquid without flow, and atoms executing diffusional hops without a concentration gradient. In general, these fluctuations are connected to behavior under external stimuli by a fluctuation-dissipation theorem. For example, the time scale $\tau$ of atomic motions in the liquid is connected to the viscosity by the DebyeStokes-Einstein relation.

Electron correlation microscopy (ECM) is a method to study equilibrium fluctuations using in situ (scanning) transmission electron microscopy (STEM) [1-3]. An ECM experiment is based on a coherent electron diffraction signal arising from the property of interest. For diffusion in solid, the signal is the strength of a Bragg beam from the atomic sites involved in the atomic hops. For liquids, the signal is a speckle arising from ordered arrangements of atoms. The intensity of that signal is measured as a function of time, $I(t)$, as it fluctuates with the underlying system, so the statistics of the intensity fluctuations reveal the temporal and spatial behavior of the system. The most common statistical descriptor is the time autocorrelation function of the intensity,

$$
g_{2}(t)=\frac{\left\langle I\left(t^{\prime}\right)\right\rangle\left\langle I\left(t^{\prime}+t\right)\right\rangle}{\left\langle I\left(t^{\prime}\right)\right\rangle^{2}}
$$

where \langle\rangle indicates averaging over the time series $t^{\prime}$. ECM offers a statistically rigorous method to measure dynamics in complex systems without the requiring imaging of the dynamics in detail.

We have applied ECM to measure fluctuations in metallic glass forming liquids as a function of temperature above the glass transition temperature, $T_{g}$ using in situ heating [1-3]. The inset in Figure 1 shows a typical nanodiffraction pattern. $g_{2}(t)$ in the plot is a measure of the lifetime of these speckles, which is in turn the lifetime of the atomic arrangements in the fluctuating liquid that give rise to them. The lines are fits to a Kohlrausch-Williams-Watt stretched exponential function,

$$
g_{2}(t)=1+A \exp \left[-2(t / \tau)^{\beta}\right]
$$

where $\tau$ is the characteristic structural relaxation time of the liquid and $\beta$ is a stretching exponent. As the temperature of liquid increases, $\tau$ decreases exponentially. Figure 2 shows spatial maps of $\tau$ derived from ECM data on $\mathrm{Pt}_{57.5} \mathrm{Cu}_{14.7} \mathrm{Ni}_{5.3} \mathrm{P}_{22.5}$ nanowires [3]. These data show that the motion of atoms in a liquid are spatially heterogeneous. At the nanometer scale, the time it takes for atoms to rearrange and swap neighbors varies by as much as an order of magnitude. This spatial variation is central to theories of the glass transition from an equilibrium supercooled liquid to the nonequilibrium solid glass. Figure 2 also shows a $\sim 1 \mathrm{~nm}$ thick surface layer with order of magnitude faster dynamics than the bulk. This fast surface layer promotes homogenous nucleation of crystals without catalytic nucleation from the surface.

Figure 3 shows practical considerations for ECM experiments. The parameter $A$ in Eq. 2 is the magnitude of the ECM signal, which increases with increasing probe coherence as shown in Figure 3(a). To obtain 
well-converged statistics, it is necessary for the time series of cover many fluctuations of the system and for each fluctuation to be sampled with several measurements. As a result, the total length of the time series must be at least $40 \tau$ as shown in Figure 3(b), and the exposure time of each pattern must be $<0.1 \tau$, as shown in Figure 3(c).

Current fast cameras have frames rates of about $1 \mathrm{kHz}$, limiting ECM to time scales $>10 \mathrm{~ms}$. Nextgeneration cameras running at $100 \mathrm{kHz}$ or faster will extend this time scale down to $<100 \mu$ s. These developments in instrumentation will enable ECM measurement on to new problems and materials [4].

\section{References:}

[1] L He et al., Microscopy and Microanalysis 21 (2015), p. 1026.

[2] P Zhang et al., Ultramicroscopy 178 (2017), p. 125.

[3] P Zhang et al., Nature Communications 9 (2018), p. 1129.

[4] Development of ECM was supported by the National Science Foundation (DMR-1807241). Development of advanced detectors and microscopy facilities are supported by the Wisconsin MRSEC (DMR1728933).

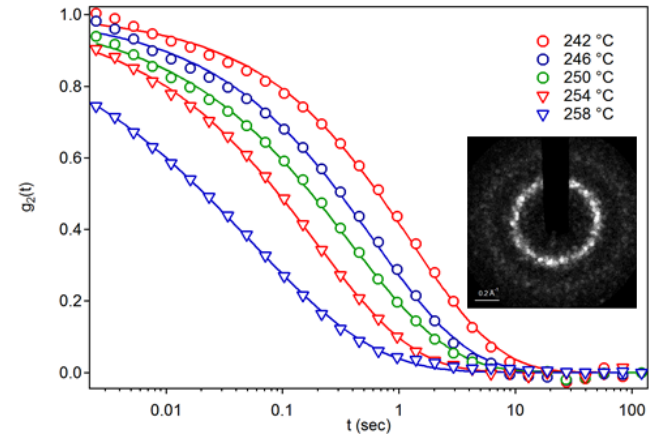

Figure 1: $\operatorname{ECM~} g_{2}(t)$ as a function of temperature from a metallic glass supercooled liquid fit to a KWW function. Inset is a typical electron nanodiffraction pattern from the liquid.
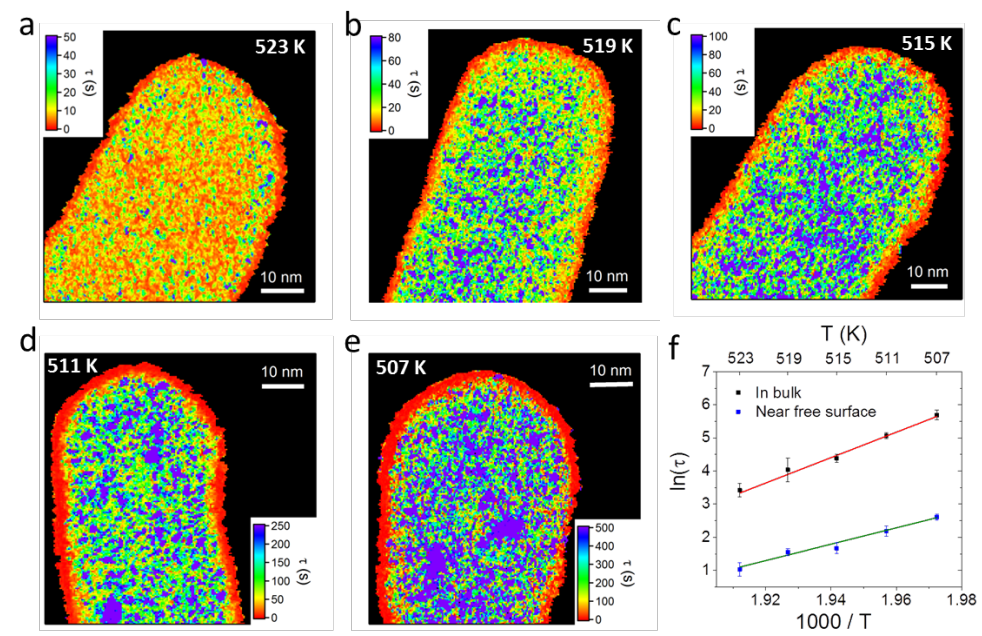

Figure 2: (a)-(e) ECM-derived maps of $\tau$ for a Pt-based metallic glass nanowire showing spatially heterogeneous dynamics within the wire and a surface layer with fast dynamics. (f) Arrhenius temperature dependence of surface and bulk $\tau$.
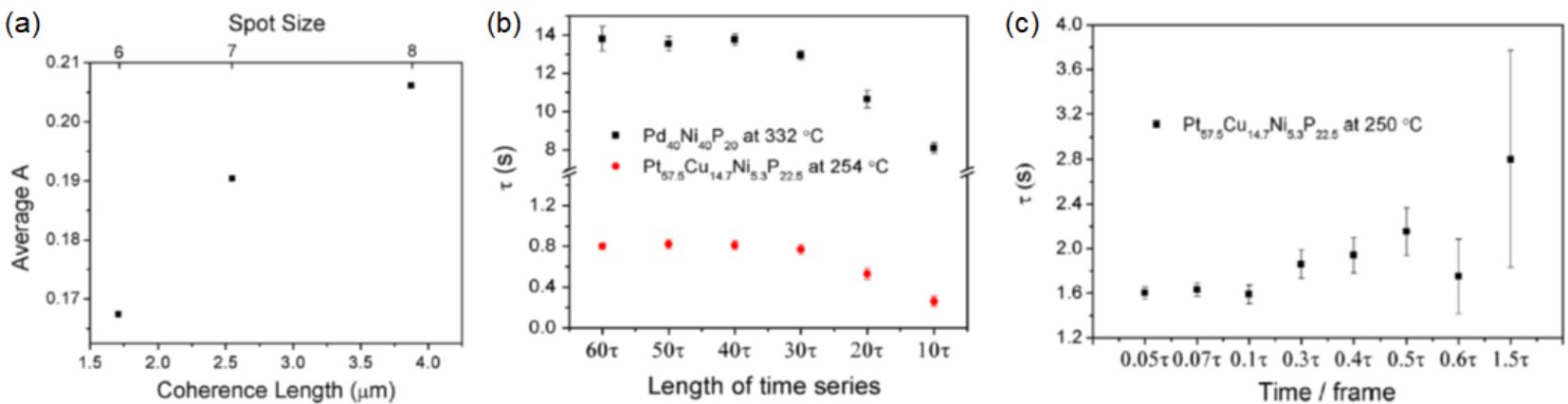

Figure 3: (a) Variation of the ECM signal with probe coherence. (b) and (c) Dependence of the structural relaxation time $\tau$ derived from ECM on the properties of the time series: (b) the total length of the time series and (c) the exposure time per diffraction pattern. 